\title{
Repolarizing macrophages improves breast cancer therapy
}

\author{
Cell Research (2017) 27:963-964. doi:10.1038/cr.2017.63; published online 21 April 2017
}

Tumor-associated macrophages (TAMs) contribute to breast cancer progression and dissemination; TAM-targeting strategies aimed at their reprogramming show promising preclinical results. In a new report Guerriero and colleagues demonstrate that a novel HDAC Class IIa inhibitor, TMP195, can reprogram monocytes and macrophages in the tumor into cells able to sustain a robust CD8 $\mathrm{T}$ cell-mediated anti-tumoral immune response.

Breast cancer is one of the most frequent types of tumors in women and despite recent advances in early diagnosis and treatment that has significantly improved outcomes, tumor resistance to chemotherapy and metastasis remain significant causes of mortality. Apart from malignant cells, breast tumors are complex evolving microenvironments consisting of resident tissue cells such as adipocytes and recruited cell types particularly those of the innate and acquired immune systems. This microenvironment plays a fundamental role in breast cancer progression to malignancy as well as resistance to therapy [1].

In breast cancers, macrophages represent a major component of this immune infiltrate. Studies in mouse models indicate that these tumor-associated macrophages (TAMs) can enhance malignancy at the primary site by stimulating angiogenesis, inducing tumor cell migration, invasion and intravasation and by suppressing anti-tumor immunity [2]. At metastatic sites metastasis-associated macrophages (MAMs) promote tumor cell extravasation, survival and subsequent growth [1]. In contrast, the infiltration of tumors by specific leukocyte cell subsets such as $\mathrm{CD} 8^{+}$ and memory T-lymphocytes has been linked with favorable outcomes in different cancers, suggesting that immune engagement can limit cancer growth and spread [1]. Indeed, in breast cancers a low ratio of macrophages to $\mathrm{CD} 8^{+}$cells predicts survival suggesting a major role for macrophages in suppressing $\mathrm{T}$ cell activity against tumors [3]. The idea of enhancing $\mathrm{T}$ cell activity in tumors is supported by the success of clinical trials with immune checkpoint inhibitors (such as anti-CTLA4, anti-PD1, and anti-PDL1) in treating melanoma and lung cancers $[4,5]$.

TAMs, despite generally being protumoral, can according to context be tumoricidal and suppress tumor growth by activating immune responses [6]. This suggests that macrophage plasticity may be therapeutically exploited to restore antitumor properties to TAMs [7]. Reprogramming methods to date, include antibody-mediated activation of costimulatory CD40, blocking of IL-10, delivery of immunostimulatory cytokines such as IL-12, or the administration of Toll-like receptor agonists or targeting intracellular signaling molecules such as PI3Kinase $\gamma$ with small-molecule inhibitors. Such strategies provide an enormous opportunity therapeutically rebalancing the microenvironment immune infiltrate from a pro-tumoral one to one that actively rejects the tumor in synergy with $\mathrm{T}$ cell-enhancing drugs such as checkpoint inhibitors.

In the paper by Guerriero et al. [8] a new approach to reprogram monocytes and macrophages into tumoricidal cells is described using TMP195, a new Class IIa Histone Acetyl Deacetylase (HDAC) inhibitors. HDACs are part of the enzymatic machinery that modifies chromatin [9] by histone de-acetylation thus changing the cell's transcriptional profile. There are 18 HDAC enzymes in mammals, subdivided into 4 main classes (Class I, II, III and IV); Class II HDAC are subdivided into Class IIA (HDAC4, HDAC5, HDAC7, and HDAC9), and Class IIB (HDAC6 and HDAC10). In the author's previous study [10] TMP195 caused changes in in vitro transcriptional profile of monocytes compared to controls, but not of $\mathrm{CD}^{+} \mathrm{T}$ and $\mathrm{B}$ cells.

In this study Guerriero et al. used the MMTV-Polyoma Middle T antigen mouse model, which develops spontaneous mammary tumors and lung metastasis [11], to demonstrate that TMP195 is able to modify the genomic profile of monocytes and macrophages, but not lymphocytes in vivo, and to limit tumor growth. After intra-peritoneal injection of TMP195 in tumor-bearing mice, the authors showed increased infiltration of $\mathrm{CD}_{11 \mathrm{~b}^{+}}$cells, but not other immune cells into the tumor. Lineage tracing of $\mathrm{CD}_{11} \mathrm{~b}^{\mathrm{Hi}} \mathrm{CSFE}^{+}$monocytes showed these $\mathrm{CD} 11 \mathrm{~b}^{+}$were recruited from the blood and differentiated into $\mathrm{CD} 45^{+} \mathrm{MHCII}^{+} \mathrm{CD} 11 \mathrm{~b}^{\text {high }} \mathrm{F} 4 / 80^{+} \mathrm{CD} 40^{+}$ macrophages. TMP195-treated animals, compared with control vehicle-treated mice, showed a lack of aberrant vasculature and reduced vessel permeability, with reduced tumor cell proliferation through an indirect mechanism. TMP195 treatment reduced primary tumor and metastatic lesion size. This effect on tumor growth was due to macrophages as their depletion by either anti-CD11b or anti-CSF1 antibody treatment abrogated the effect of TMP195.

To test the role of the adaptive immune system in the observed TMP195 tumor inhibition, Guerriero et al. trans- 


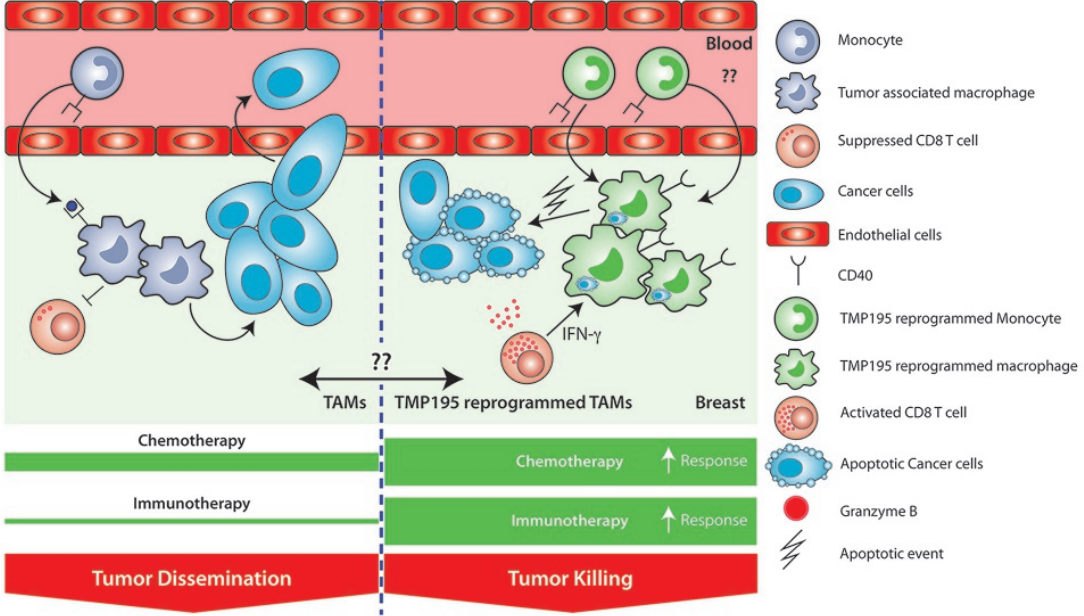

Figure 1 Reprogramming of monocytes and macrophages by HDAC inhibitor TMP195 inhibits mammary tumor progression. Schematic representation of monocyte recruitment in the tumor site in the absence (left panel) or presence (right panel) of TMP195. Macrophages promote tumor dissemination in the absence of TMP195 and PyMT tumors respond to chemotherapy modestly but not immunotherapy (left panel, green bars), whereas they become anti-tumoral and promote tumor killing when the reprogramming agent is present and TMP195 treatment make tumors more responsive to chemotherapy and immunotherapy (right panel, green bars). Thickness of green bar indicates response to therapy. Question marks represent open questions to be investigated, i.e., the recruitment mechanism of reprogrammed monocytes to get access to the tumor site, and the potential rebound effect when the reprogramming agent is removed.

planted autochthonous tumor pieces into Foxn $1^{n u}$ mice lacking $\mathrm{T}$ cells, or wildtype (WT) control mice and measured tumor burden after TMP195 treatment. Only in WT mice was TMP195 able to reduce tumor growth while additional experiments depleting CD8 or CD4 T cells demonstrated that active CD8 T cells are required for TMP195 to show tumor inhibition. Moreover, the proportion of $\mathrm{CD} 8 \mathrm{~T}$ cells expressing granzyme B was also increased upon TMP195 treatment. Furthermore, the authors showed that TMP195 was not able to reduce tumor burden in the absence of IFN $\gamma$ mainly produced by $\mathrm{CD} 8 \mathrm{~T}$ cells. Finally, the authors showed that TMP195 in combination with standard chemotherapy (carboplatin or paclitaxel) was significantly more effective in reducing tumor size than monotherapy. In addition, TMP195 used in combination with anti-PD1 antibodies that enhance $T$ cell action showed a significant synergistic effect compared with anti-PD1 treatment alone, which on its own was unable to decrease tumor burden.

In conclusion, this is the first study showing that Class II HDAC inhibitors can selectively reprogram monocytes and macrophages in the tumor; the reprogramming activates a robust anti-tumor immune response, mainly mediated by macrophages, CD8 T cells and IFN $\gamma$, that reduce both primary and metastatic tumor burden. This treatment also synergizes with chemo- and immuno-therapy (Figure 1). Thus this study opens new clinical applications for future TAM reprogramming in breast cancer. It contrasts with current TAM depletion strategies, e.g., by the inhibition of the colony stimulating factor 1 receptor (CSF1-R) that has showed promising results in diffuse-type giant cell tumors [12] but is likely to be toxic in many cases as this treatment ablates most macrophages. Nevertheless, despite its apparent advantages several questions about the mechanism of action of these new HDAC inhibitors remain open. For example, the authors showed increased CD11 $\mathrm{b}^{+}$ cells infiltrating the tumors, but they did not identify which stimuli and receptors were involved in this recruitment or the stability of the reprogrammed phenotype upon long-term treatment. They also did not examine other tissues for toxicity nor did they study what happens after cessation of treatment (Figure 1). Caution is needed as recent studies showed that monocyte recruitment after stopping anti-CCR2 treatment could have dangerous rebound effects that promote metastasis [13]. Similarly, it will be important to investigate the effects of TMP195 on other myeloid cells, such as neutrophils, to avoid compensatory mechanism that could impair the efficacy of a prolonged macrophage reprogramming in the tumor.

Luca Cassetta ${ }^{1}$, Jeffrey W Pollard ${ }^{1,2, *}$

${ }^{\prime} M R C$ Centre for Reproductive Health, Queen's Medical Research Institute, The University of Edinburgh, Edinburgh EH16 4TJ, UK; ${ }^{2}$ Department of Developmental and Molecular Biology, Albert Einstein College of Medicine, New York, New York 10461, USA

Correspondence: Jeffrey W Pollard

E-mail: Jeff.Pollard@ed.ac.uk

\section{References}

1 Noy R, Pollard JW. Immunity 2014; 41:49-61.

2 Kitamura T, Qian BZ, Pollard JW. Nat Rev Immun 2015; 15:73-86.

3 Ruffell B, Au A, Rugo HS, et al. Proc Natl Acad Sci USA 2012; 109:2796-2801.

4 Hodi FS, O'Day SJ, McDermott DF, et al. $N$ Engl J Med 2010; 363:711-723.

5 Brahmer J, Reckamp KL, Baas P, et al. $N$ Engl J Med 2015; 373:123-135.

6 Wynn TA, Chawla A, Pollard JW. Nature 2013; 496:445-455

7 Williams CB, Yeh ES, Soloff AC. NPJ Breast Cancer 2016; 2. pii: 15025.

8 Guerriero JL, Sotayo A, Ponichtera HE, et al. Nature 2017; 543:428-432.

9 Das Gupta K, Shakespear MR, Iyer A, et al. Clin Transl Immunology 2016; 5:e62.

10 Lobera M, Madauss KP, Pohlhaus DT, et al. Nat Chem Biol 2013; 9:319-325.

11 Lin EY, Jones JG, Li P, et al. Am J Pathol 2003; 163:2113-2126.

12 Ries CH, Cannarile MA, Hoves S, et al. Cancer Cell 2014; 25:846-859.

13 Bonapace L, Coissieux MM, Wyckoff J, et al. Nature 2014; 515:130-133. 